\title{
HALO flight test with instrumented under-wing stores for aeroelastic and load measurements in the DLR project iLOADS
}

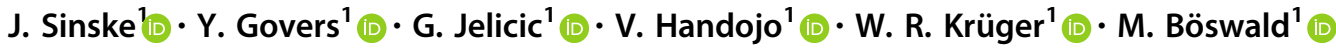

Received: 8 March 2017 / Revised: 29 August 2017 / Accepted: 15 February 2018/ Published online: 27 February 2018

(c) The Author(s) 2018. This article is an open access publication

\begin{abstract}
HALO (High Altitude and Long Range Research Aircraft), the atmospheric research aircraft of the German Aerospace Center (DLR), can be equipped with under-wing stores at different wing positions to transport scientific instruments for atmospheric research. The particle measurement system (PMS) carrier is such an external store which can carry three instruments at the same time per wing. Any modifications on an aircraft must be tested numerically and experimentally to ensure the structural integrity of the aircraft for all flight conditions. Load and flutter analyses can be validated with flight test data. For flight test, the aircraft and the under-wing stores of HALO must be equipped with acceleration and strain sensors. To reduce flight test time it is necessary to make quick decisions during the flight test. Therefore the DLR Institute of Aeroelasticity in Göttingen has developed a real-time analysis procedure for online identification of modal parameters like eigenfrequencies, damping ratios and mode shapes. These parameters vary with flight conditions and are necessary to analyse the aeroelastic stability of the system. The department of loads analysis and aeroelastic design and the department of structural dynamics and system identification have tested the newly developed procedure in 14 flight hours on the HALO. A network of three distributed data acquisition modules enabled the recording of the flight test instrumentation with 51 accelerometers and 16 strain gauge bridges. The measured data were distributed online on several computers where the newly developed software was implemented, allowing an instantaneous analysis of the structural dynamics behaviour and loads in flight. This paper provides an overview of the conducted flight vibration tests with HALO. It also shows the capability of the newly developed online monitoring system for aeroelastic identification.
\end{abstract}

Keywords Flight vibration test · Operational modal analysis · Automated online aeroelastic identification

\section{Introduction}

The aeroelastic (flutter) stability of aircraft prototypes and major modifications on existing aircrafts is calculated on the basis of a finite element model during design phase. The structural model and the corresponding flutter analyses need to be validated with tests on ground and in flight after completion of the aircraft. During experimental validation the structural dynamic properties (eigenfrequencies, modal damping ratios and mode shapes) of the aircraft are firstly determined on ground in a so called ground vibration test (GVT). Later on the structural dynamic properties are identified from various flight states (altitude and speed) to ensure the flutter stability.

\section{J. Sinske}

Julian.Sinske@DLR.de

1 German Aerospace Center (DLR), Institute of Aeroelasticity, Göttingen, Germany
Conventional flight vibration test (FVT) are similar to ground vibration tests [1-4]. Forces are introduced into the structure via movement of the control surfaces, e.g. with pulses or controlled swept sine excitation or even external exciters with mass unbalances or exciters with oscillating vanes. Defined movements of the control surfaces are only possible via an electronic flight control system (EFCS) or a fly by wire (FBW) control and external exciters installed at the wing tips, are quite expensive and not easy to install. Also atmospheric turbulence gives a certain level of broadband excitation on the aircraft structure, but so far it has not been used for modal identification.

In the context of the iLOADS [5] flight test campaign, modal identification methods, e.g. Operational Modal Analysis (OMA), as well as methods for load determination are tested by DLR for the first time in a flight vibration test. OMA, which is an output-only modal analysis method, offers the possibility to directly identify the modal 
parameters (eigenfrequencies, damping ratios and mode shapes) without the knowledge of the excitation forces. It is based on the assumption that only stochastic excitation forces are applied to a structure. During DLR's internal ALLEGRA project (2012-2016) the DLR Institute of Aeroelasticity optimised the computational highly demanding methods to such an extent that they reached nearly real-time capability [6-8]. The developed system has already shown real-time aeroelastic identification capability within several wind tunnel testing campaigns in the transonic wind tunnel Göttingen (TWG) and the cryogenic wind tunnel in cologne (KKK) from German Dutch Wind Tunnels (DNW) $[6,9,10]$.

HALO $^{1}$ (Fig. 1) is already equipped with a measurement system called BAHAMAS that serves instruments for atmospheric research. Nevertheless, appropriate flight test instrumentation (FTI) suitable for vibration tests and loads identification was not available and had to be planned for the iLOADS campaign. This FTI consisted of sensors, cabling, a measurement system as well as a data acquisition PC and four analysis PCs. To analyse the measured time signals efficiently in flight a network was established between the PCs for live data distribution.

The online monitoring software, which was developed at the DLR Institute of Aeroelasticity, has been used for the first time during a flight test to directly analyse the acceleration and strain time data. OMA methods have been examined for its flight test ability on large aircraft structures and will be presented in this paper. During the first part of a longer flight test campaign several forms of vibration excitation have been tested to qualify suitable excitation techniques for the identification of the structural dynamic properties.

The second part of the flight test campaign focused on the loads between the under-wing stores and the wings, which act during flight manoeuvres. The measured manoeuvre loads should be validated with simulation data of the numerical model.

The flight test campaign consisted of five flights in total. Each flight had duration between 90 and $180 \mathrm{~min}$. This results in total to flight duration of approximately $14 \mathrm{~h}$. Test flights number one, three, four and five mainly focused on in flight vibration analysis. During the second flight the focus was put on load measurement, where load manoeuvres, such as roll- and pull-up manoeuvres were flown at seven different altitudes and three different flight speeds. For the flight vibration tests several altitudes with different flight speeds have been flown to identify the changes in the structural dynamical behaviour of the aircraft. At each measurement point, the flight speed and the

\footnotetext{
${ }^{1}$ http://www.dlr.de/dlr/en/desktopdefault.aspx/tabid-10203/339_ read-268/\#/gallery/136.
}

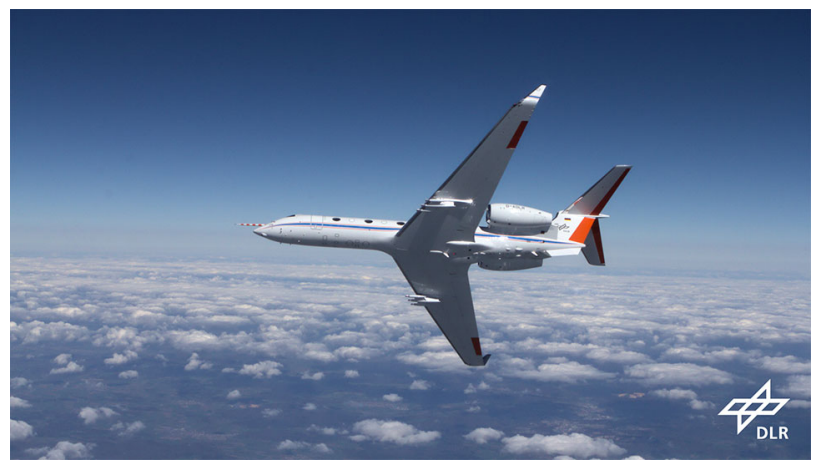

Fig. 1 HALO during flight test with under-wing stores

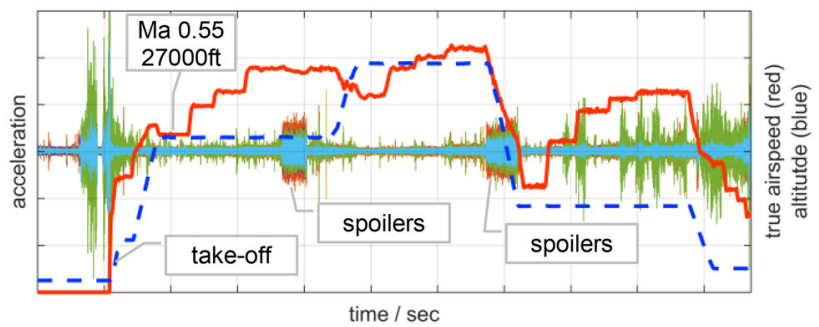

Fig. 2 Time history of acceleration signals with flight altitude and flight speed

altitudes have been kept constant for about 5 min. Within this timeframe, the automatic OMA method provided quasi real-time results of the changes in the vibration behaviour.

A typical flight profile is shown in Fig. 2. The blue curve shows the flown altitude profile and the red curve shows the true airspeed. Some acceleration signals of a typical flight are plotted behind the two parameter variations and provide different excitation levels, e.g. by extending the spoilers, impulse inputs via the control surfaces or the Take off at the beginning of the time data plot.

\section{Flight test instrumentation}

The flight test instrumentation, which was developed and purchased for this flight test, consisted of acceleration sensors, strain gauges and the necessary cabling. These sensors were measured with a compact and robust National Instruments cDAQ measurement system. This measurement system was successfully validated in advance for the high requirements of the flight test. Time data measured with the data acquisition system was stored on the same PC and at the same time distributed inside the aircraft to the analysis PCs via a data stream over the network.

One of the requirements for the data acquisition system was a placement in the under-wing stores, which is a cylinder of $15 \mathrm{~cm}$ diameter. The PMS Carrier already provided existing network connections, which transferred 


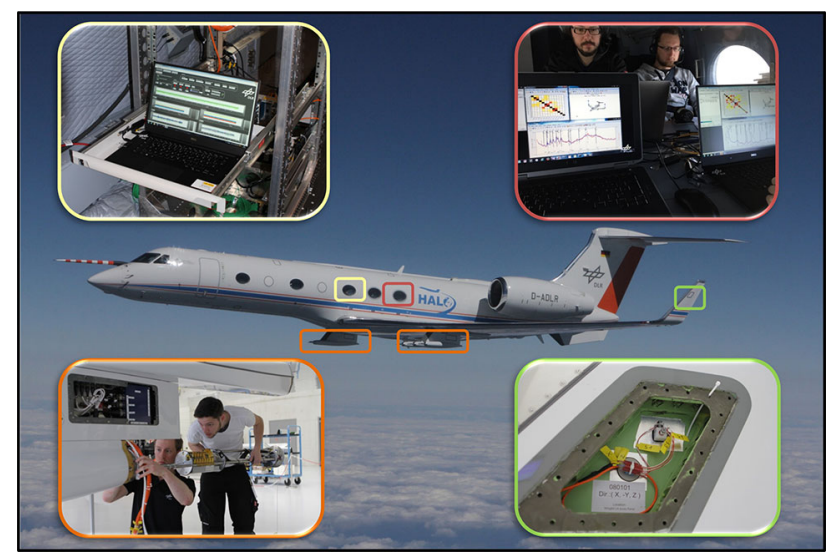

Fig. 3 Measurement and analysis chain

the data into the fuselage. Also power supply was available for the individual units of the measurement system.

The complete measurement chain is shown in Fig. 3. The acceleration/strain sensors (green) are measured via the NI measurement system (orange) and the data acquisition PC (yellow). Inside the cabin the data stream was directly transferred to the analysis PCs for online analysis (red).

\subsection{Sensor positions}

HALO and the attached PMS Carrier were instrumented with a total of 67 sensors including 51 piezoelectric accelerometers (see Fig. 4) for flight vibration testing and 16 strain gauges for the determination of loads in flight.

The acceleration sensor positions were concentrated on the PMS Carrier to resolve the local vibration behaviour of the PMS Carrier. The global vibration behaviour of the aircraft could be monitored with sensors distributed in the fuselage, tail plane and wing tips.

16 strain gauge bridges were placed on the Hanger Beam between the PMS carrier and the wing (see Fig. 5) to determine the loads between PMS carrier and the hard points at the wing.

\subsection{Rugged and open DAQ for flight testing with real-time capabilities}

A new measurement unit had to be selected and procured for the flight test. An important criterion for this was the possibility to access the measured time data online and transfer it to in-House analysis software for real-time processing. Furthermore, the measuring system needed to be compact to fit it in the limited space of the PMS carriers. A further request was a distributed setup of measurement units with a common time base and synchronized sampling.

The distributed setup of the measurement units inside and outside the pressurized cabin required an especially

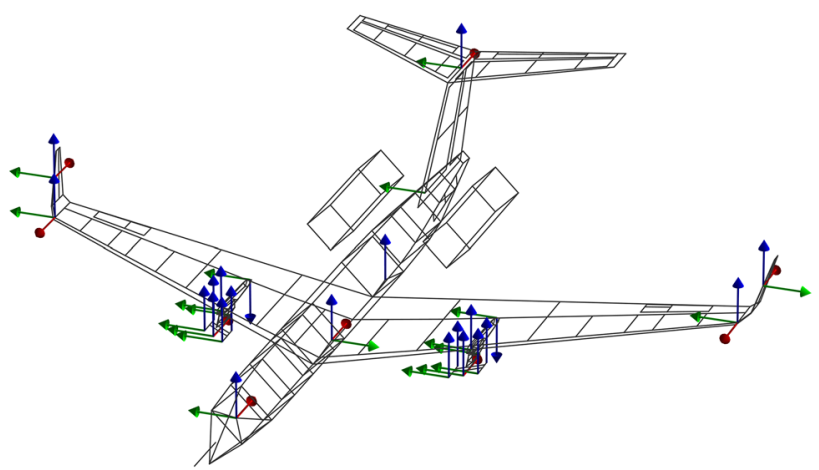

Fig. 4 Sensor plan of the accelerometers

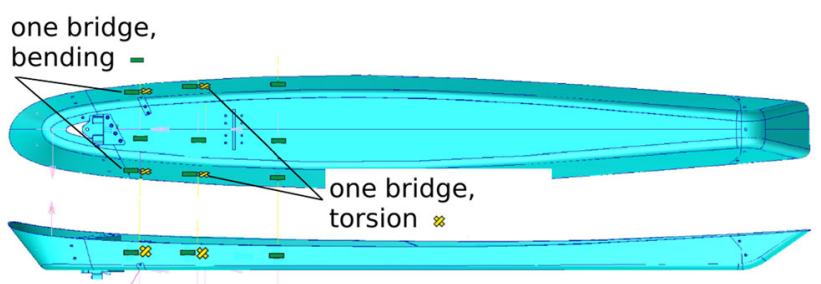

Fig. 5 Strain gauge positions on the hanger beam
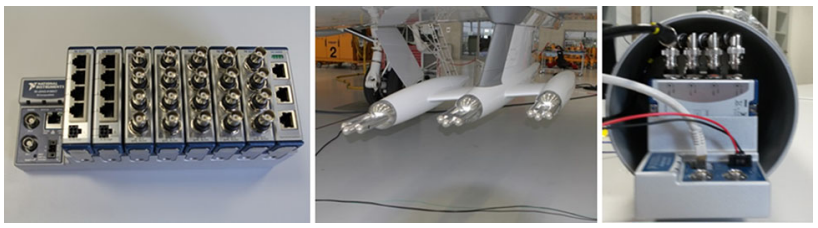

Fig. 6 Small and robust measurement unit for flight testing

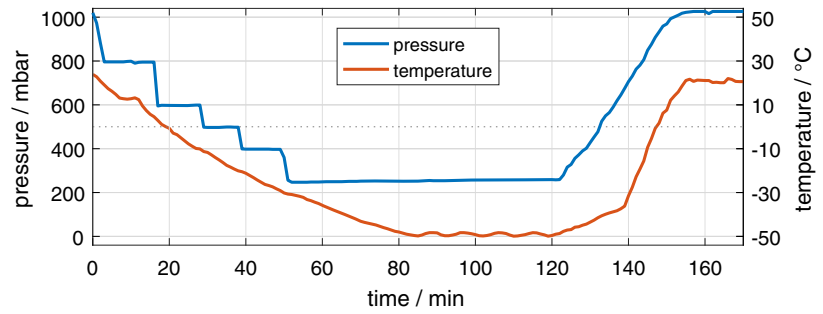

Fig. 7 Qualification test of DAQ in climate chamber

robust system. The system had to work reliably at low temperatures and low air pressure in high altitudes. The selected NI cDAQ measuring system (see Fig. 6), which is very compact and allows online access to the time data, is specified by the manufacturer only up to an altitude of $5000 \mathrm{~m}$ and a temperature down to $-40{ }^{\circ} \mathrm{C}$. This limited specification required a climatic chamber test in which the requirements of a flight profile were simulated, as it would occur during the targeted flight tests.

During this climatic chamber test (see Fig. 7), the measuring system together with the sensors was exposed to a pressure of less than 250 mbar, which simulated an 
altitude of approx. 11,500 $\mathrm{m}$ and a temperature of below $-50{ }^{\circ} \mathrm{C}$. The measurement system worked perfectly during this 3-h test and showed no drifts in the measured signals, no errors or any failure.

A further request for the flight test certification on the measuring system was a test of electromagnetic compatibility as part of an EMC test in an EMC chamber and later in the installed state on the entire aircraft. These tests intended to exclude the measurement electronics from influencing important components of the aircraft such as board electronics or radio communication.

The suitability of the relatively inexpensive Ni-cDAQ measuring system for airborne test conditions has been successfully demonstrated both in the climatic chamber test and in the EMC test. The results gained during these preliminary tests were also confirmed during the flight test campaign under real conditions, where the measuring system worked without any failure over the entire duration.

\subsection{Distributed setup for measurement system and data network}

The complete setup of the distributed measuring system (see Fig. 8) consisted of three individual NI-cDAQ chassis. The master acquisition unit of the three chassis (NI-cDAQ Master) was placed in the pressurized cabin of the aircraft fuselage next to the data acquisition PC. The other two chassis (NI cDAQ Slave 1 and 2), were placed, respectively, in the left and right PMS Carrier under the wing. The two slave chassis were connected to the master chassis in the fuselage with only two ethernet cables for synchronization and data transfer of the digitized measurement signals. This design of the measuring system considerably reduced the length of the analogue signal routing and was less prone to interference, e.g. by radio signals or the on board electronics. A further advantage was a significant reduction in the installation effort, since time was limited during installation phase before the flight test.

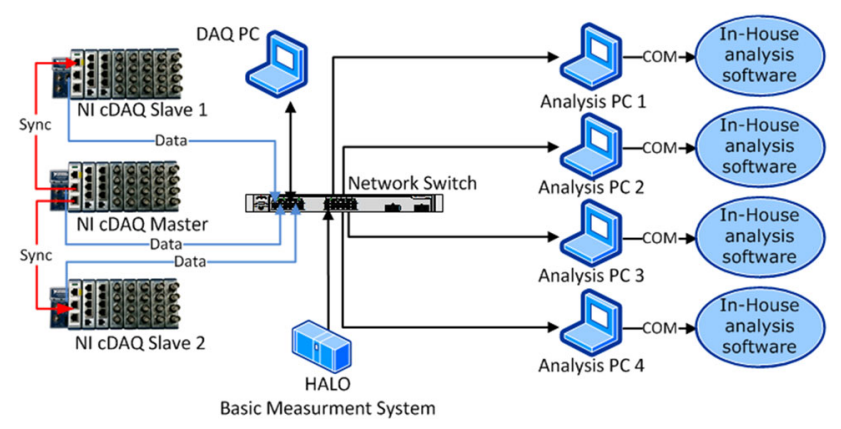

Fig. 8 Data network for the online distribution of the measured data to the analysis PCs
The time data signals were recorded with all three chassis and stored on the data acquisition PC (DAQ PC). During acquisition the measurement signals were displayed on the screen of the DAQ-PC for verification.

Time stamped flight parameters (altitudes, airspeed, pitch angle, etc.) were transmitted from the basic measurement system (BAHAMAS) via network stream to the DAQ-PC.

The DAQ-PC distributed the data directly over a network, therefore, it was possible to perform modal analysis and loads identification simultaneously in real time on several analysis PCs. Besides the access of the client PCs for post processing was controlled by the DAQ-PC.

\section{Flight vibration test}

For the flight vibration tests it was mandatory to keep the flight state constant which was indicated by parameters like altitude and speed over a certain period of time. To measure the dynamic behaviour of the aircraft at different flight states the aircraft was haled at a defined altitude, then the flight speed was changed to the next level and kept constant for a defined period of time. This procedure is shown in the flight profile of the flight parameters in Fig. 2. In one of the test cases the aircraft maintained the flight level at an altitude of 27,000 ft and the flight speed of Mach 0.55 was kept constant for about $5 \mathrm{~min}$. During this stable flight state the measured acceleration signals are automatically analysed by the DLR developed online monitoring method and the identified modal parameters for this test point are stored into a database. Three further flight speeds (up to Mach 0.82) were analysed at this altitude and identified modal parameters at these measuring points were stored in a database. Subsequently, the procedure was repeated on the altitudes 40,000 and 12,000 ft. Already during the flight, the modal parameters could be retrieved from the database and the changes of the dynamic behaviour as a function of flight speed and altitude could be analysed and visualized. In the following three flight vibration tests, additional flight speeds and altitudes were considered. With this data a test matrix was built up, which covered the dynamic properties of HALO over the entire flight envelope.

\subsection{FTI qualification test with the method of Taxi Vibration Test}

In contrast to a conventional Ground Vibration Test (GVT) which employs artificial excitation, natural excitation on run- or taxiways as shown in Fig. 9 is used in taxi vibration testing. GVT of aircraft prototypes is one of the most challenging tasks in vibration testing. For an aircraft of the size of an A320 or B737 a high channel count data 


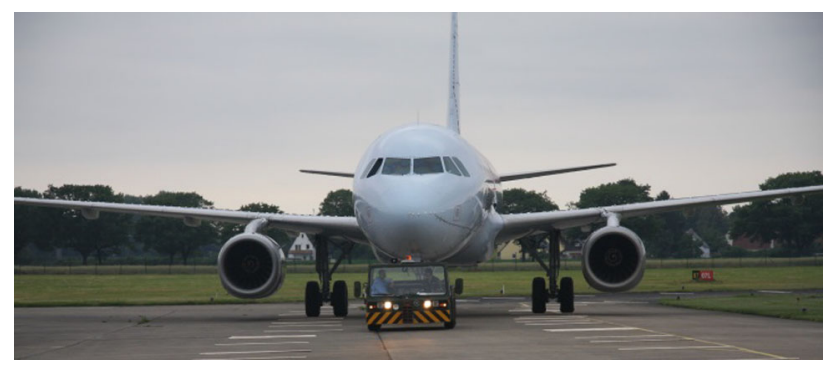

Fig. 9 ATRA Taxi Vibration Test, Manching 2009

acquisition system is needed that can acquire several hundred of accelerometers $(\sim 600)$ simultaneously. About 15-20 electro-dynamic exciters with maximum force levels in the range of $1000 \mathrm{~N}$ and long coil stroke are required. Especially the excitation of the aircraft at flexible excitation points such as wing tips or winglets requires a lot of experience not to run into the limits of the shaker. Besides all the specific equipment an experienced team must be available to run the test efficiently in terms of time and quality of the results.

Before the first flight a taxi vibration test (TVT) [11-14] was performed to verify the installed sensors and measuring systems under operating conditions. The TVT method has been developed by the Institute of Aeroelasticity and was already applied on several aircraft. Figure 9 shows the DLR aircraft ATRA during the Taxi Vibration Test in Manching 2009 [14].

The TVT method is conducted by simply towing an aircraft over runway or taxiway at constant speed, e.g. 5, 10 or $15 \mathrm{~km} / \mathrm{h}$. This method provides enough vibration amplitude on the installed acceleration sensors to enable output-only modal Analysis. Proceeding this way, it is possible to identify several eigenfrequencies, modal damping ratios and eigenvectors of the structure.

With the TVT on HALO (see Fig. 10) the flight test setup could be verified without the need of a ground vibration test and a modal reference data basis could be created.

During TVT a continuous stream of time data was recorded for duration of roughly $1 \mathrm{~h}$. It is beneficial for a

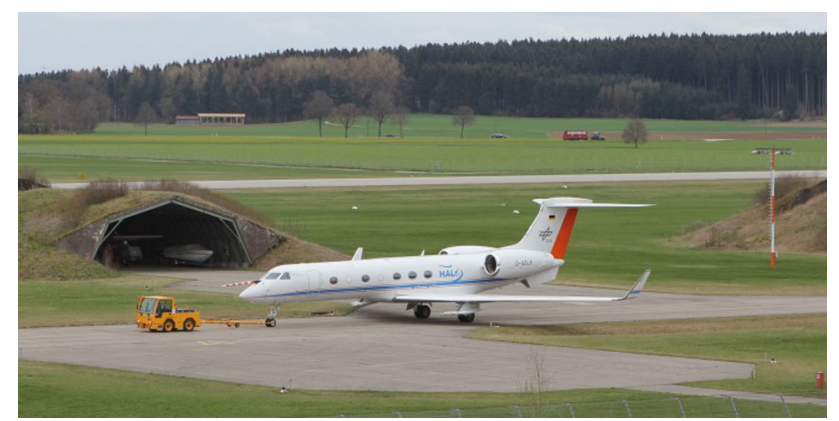

Fig. 10 HALO Taxi Vibration Test, Oberpfaffenhofen 2016 high quality TVT results to maintain a constant level of excitation on the whole structure. Generally the level of excitation is dependent on the driving speed of the tractor and also on the roughness of the ground or pavement. Also driving at higher speeds over the same surface roughness will lead to a shift of the excited frequency range towards higher frequencies at the cost of lowering the excitation levels at lower frequencies.

HALO was towed over landing and taxi ways for $60 \mathrm{~min}$ at Oberpfaffenhofen airport. Figure 11 shows time histories that were already divided into several pieces. They describe where the aircraft was towed, on runway, taxiway or in between in a turn.

A nice representation of the different levels of excitation is given by statistical analysis of the time domain data in Fig. 12, which shows the 1st-4th sample moment of the acceleration time histories. 3rd and 4th moment of the time data signals show special features of the data. The skewness (3rd moment) shows, if nonlinear effects in the structure are activated. There is also a significant difference between the normally distributed acceleration histories on the runway and acceleration histories on the bumpy taxiway. If we consider an aircraft rolling on uneven pavement the biggest source of non-linearity originates from the influence of the landing gear shock absorbers.

Figure 12 also shows that the data from the runway seems to be most consistent.

The analysis of the statistical moments for the taxi vibration test time data also allows for immediate detection of faulty sensors, which is also presented by grey curves in Fig. 12. The signals show significant deviation from all others for the 3rd and 4th moment. Two sensors were identified that have fallen off before we went for the first flight test.

A further indicator for the consistency of the acquired time data is given by the spectrogram presented in Fig. 13. Especially during turns the different behaviour was visible

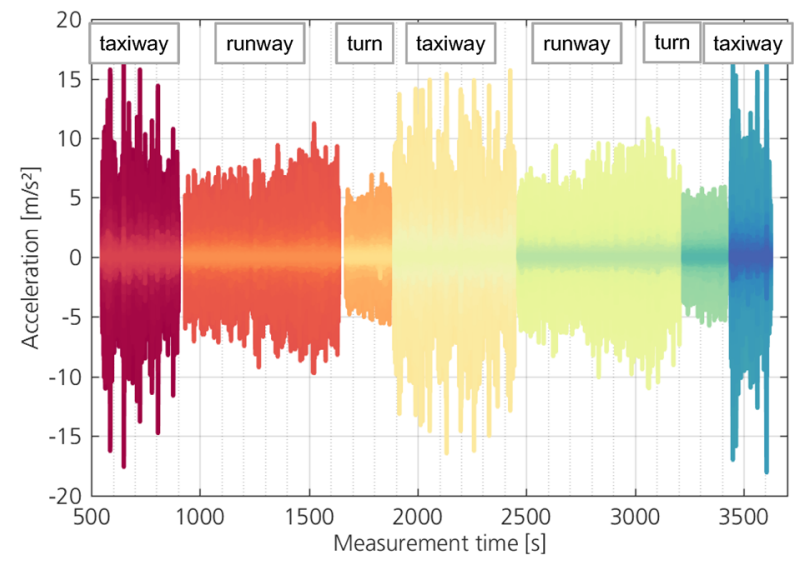

Fig. 11 Acceleration time histories from taxi vibration test 

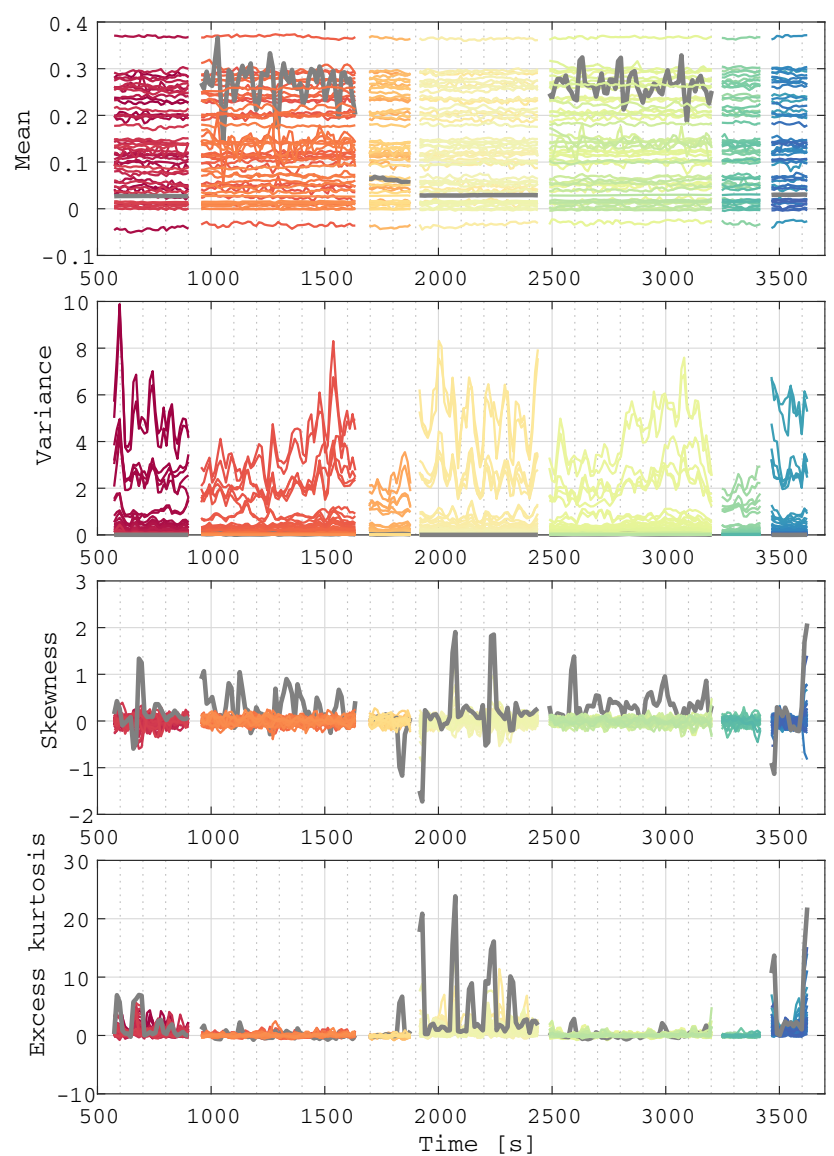

Fig. 12 Sample central moments of acquired accelerometer histories during taxi

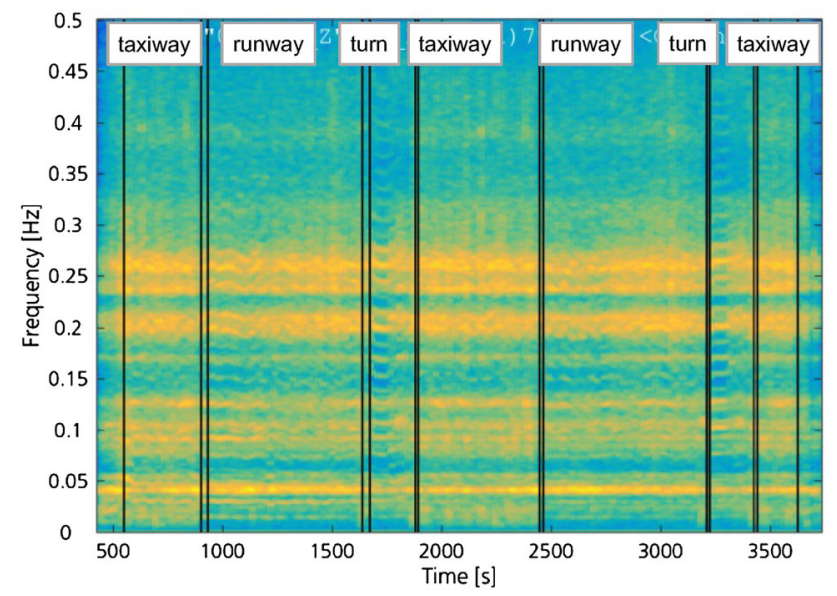

Fig. 13 Spectrogram of TVT data

which can also be referred to the variation of speed during turning. The most consistent excitation level is again visible for the runway sections.

For further evaluation the runway dataset from seconds 1000-1600 has been chosen. The time data was used for output-only analysis. Cross-power spectral density (CPSD)

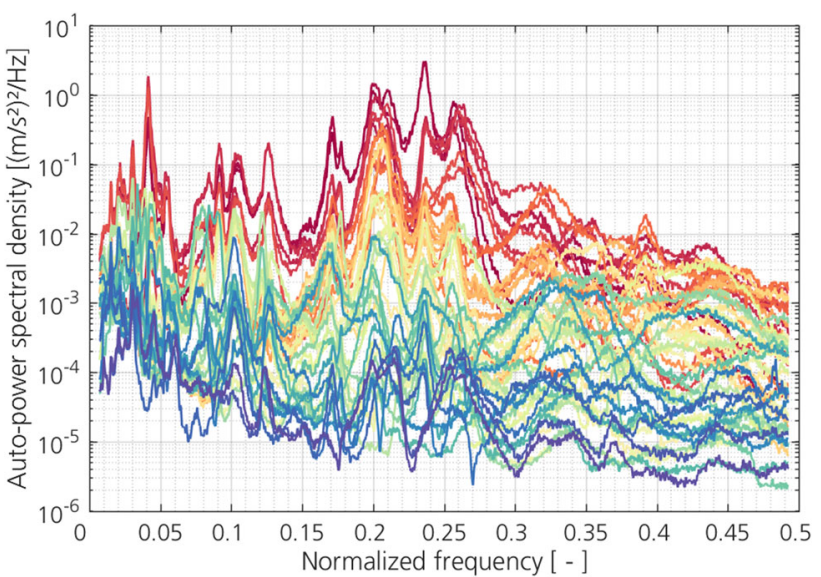

Fig. 14 Auto-power spectral densities of TVT data

functions are calculated referencing on two sensors. The sensors are symmetrically installed in $y$-direction and placed at the left and right wing external stores. The crosspower spectral densities are shown in Fig. 14. Several peaks at distinct frequencies are visible which already indicate eigenfrequencies of the structure.

From CPSD's stabilization diagrams are calculated. The frequency-domain LSCF (least squares complex frequency domain) algorithm employed for operational modal analysis requires cross-power spectral densities.

The measured time data was not processed to frequency response functions as usually but to cross-power spectra. These cross-power spectra generally describe the dynamic response of the aircraft in dependence of a reference, which can be, e.g. the signal of a predefined sensor. From the calculated spectra it was possible to identify modal parameters.

A selection of the mode shapes identified in the taxi vibration test is shown in Fig. 15. The selection includes rigid body modes (a, b) and symmetric (c) as well as antisymmetric elastic modes (d-f) and local modes ( $g, h)$ unsymmetrical modes are, e.g. from the mounted PMS carriers $(\mathrm{g})$.

\subsection{Automatic modal analysis in flight}

An essential part of the flight test campaign was the validation of the automatic output-only modal analysis method in real-time.

The graphical user interface (GUI) of the used software is shown in Fig. 16. The software was able to execute the necessary steps for evaluating the TVT or FVT data within less than $3 \mathrm{~s}$. Therefore, time data was processed into cross-power spectral densities with pre-defined signal processing parameter settings, modes were calculated and selected automatically. The identified modal datasets were written into a database together with various aircraft and 


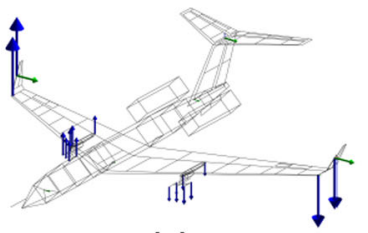

(a)

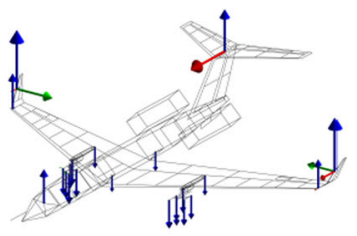

(c)

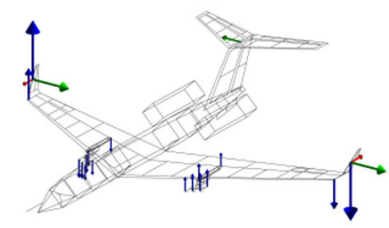

(e)

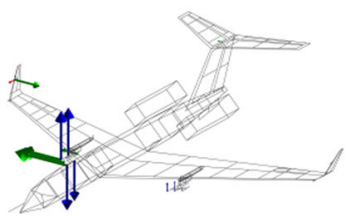

(g)

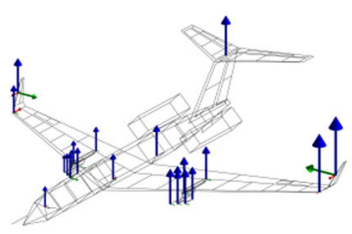

(b)

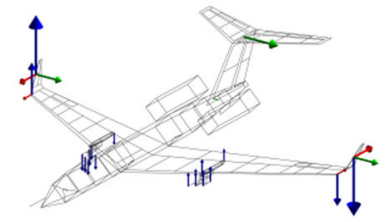

(d)

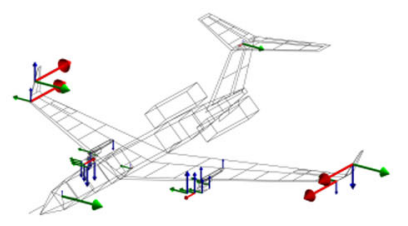

(f)

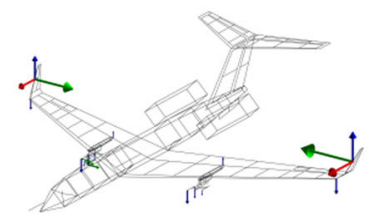

(h)
Fig. 15 Identified modes from the TVT

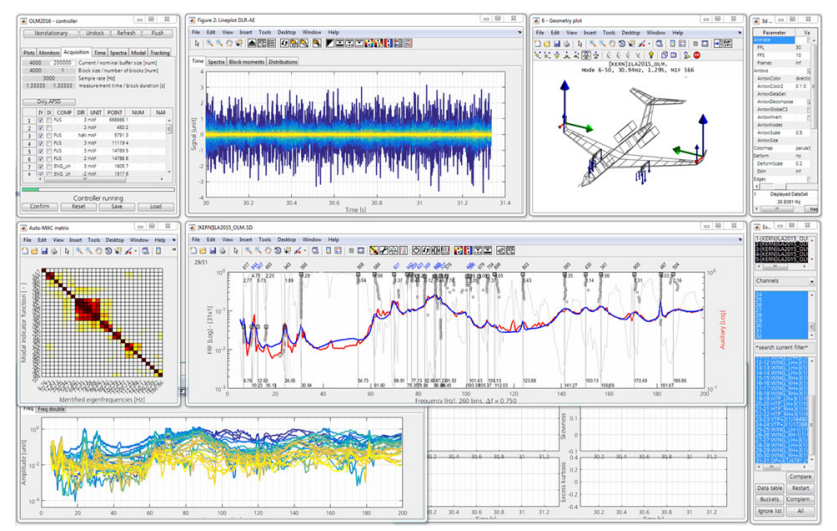

Fig. 16 GUI of modal analysis software with generic data

environmental parameters, which were provided from the BUS system of the aircraft. In this way, changes in the eigenfrequency and modal damping with altitude and Mach number could be displayed and interpreted. Eigenfrequency and modal damping ratios have to be assigned to an explicit mode. The values are automatically sorted via the MACXP (Modal Assurance Criterion eXPanded) [15] matrix. Thus, it is ensured that observed changes can be assigned to the "same" mode.
The high computing effort could be handled on Core-i7 laptops. For the analysis of the data three computers were available in parallel so that different settings could be used and tested simultaneously.

\subsection{Comparison of various excitation techniques in flight}

Various types of excitation techniques of the aircraft structure were analysed during the flight vibration tests. It should be determined, which kind of excitation was particularly suitable for the output-only modal analysis procedures. The question was, for example, whether less or highly turbulent flow is better to achieve more accurate analysis results. One form of additional excitation is a pulse-shaped control input from the pilot on the aileron and the elevator. Herewith, higher frequency modes should be better excited. Some selected measurement points are repeated with and without spoilers. The extended spoilers generate significantly higher vibration amplitudes (see Fig. 2).

Another promising method was tested in the fourth flight. This flight is performed together with another aircraft (DLR Falcon) to excite the HALO with wake vortices of another aircraft. Therefore, the Falcon is positioned at two altitudes and different speeds in front of the HALO. This procedure is shown in Fig. 17. To avoid unstable and unsafe flight conditions the HALO approached slowly from a safe distance until the turbulences were sufficiently strong. This state was kept constant for about five minutes to perform the online modal analysis. Table 1 summarizes the measurement points acquired during flight vibration testing that are presented in this document. Modal analysis performed on these datasets is presented in [8].

Figure 18 displays all acceleration histories during constant level flight at $27,000 \mathrm{ft}$ with pure atmospheric turbulence excitation (MP11 $\div$ MP14) and with air brakes (spoilers) active (MP15) at Mach speeds approximately constant for each measurement point. It is clear that the excitation level increases significantly when air brakes are deployed. The skewness and kurtosis of multiple data blocks shown in Fig. 19 display sample distributions that are mostly symmetric, but with heavier tails at lower

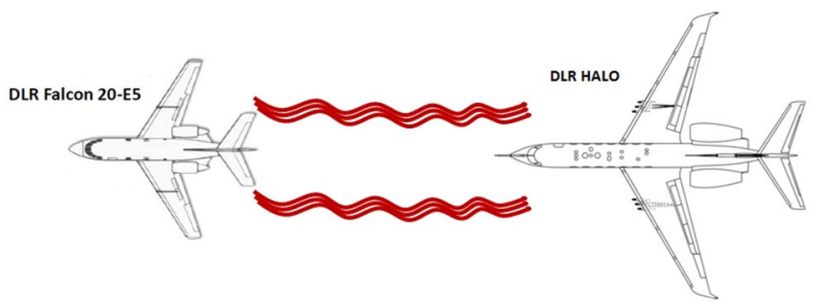

Fig. 17 HALO in the turbulent flow of the ahead flying DLR Falcon 
Table 1 Presented flight vibration test measurement points; flight altitude is 27,000 $\mathrm{ft}$ for all

\begin{tabular}{llll}
\hline$\#$ & Mach & Comment & Duration (s) \\
\hline MP11 & 0.55 & Constant heading, pure turbulence excitation & 304.7 \\
MP12 & 0.65 & Constant heading, pure turbulence excitation & 306.3 \\
MP13 & 0.70 & Constant heading, pure turbulence excitation & 324.4 \\
MP14 & 0.78 & Constant heading, pure turbulence excitation & 310.3 \\
MP15 & 0.77 & Constant heading, air brakes active & 304.7 \\
MP21 & 0.70 & Stochastic elevator/aileron excitation & 434.5 \\
MP41 & 0.70 & Flying in preceding airplane's wake & 375.0 \\
\hline
\end{tabular}

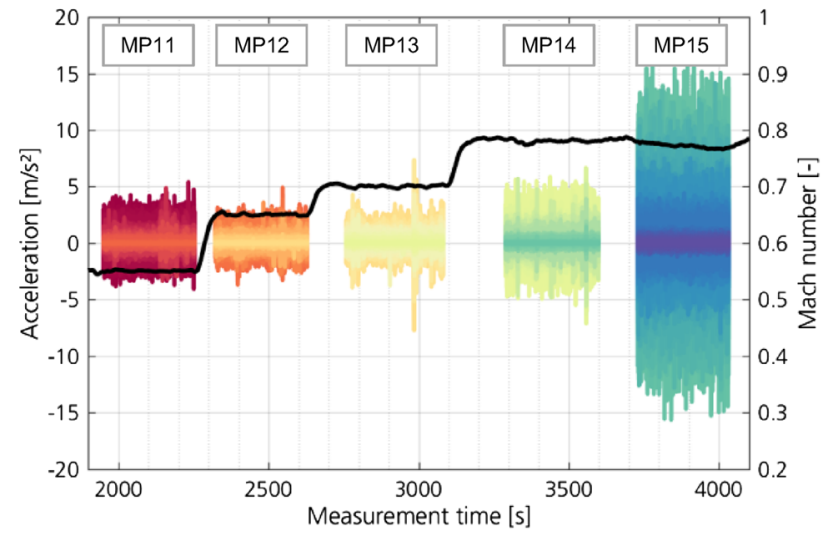

Fig. 18 Time data of all accelerometers (MP11 $\div$ MP15)

excitation levels, which suggests a higher susceptibility to outliers caused by transients, impulsive events and course corrections. When air brakes are deployed the resulting turbulence provides highly normally-distributed acceleration histories due to higher excitation level. The autopower spectral densities estimated in Fig. 20 demonstrate how the excitation level due to turbulence (MP11 - MP14) rises monotonically in the current Mach range. Of particular interest is the fact that air brakes provide a tenfold acceleration response increase in the higher frequency range (in RMS terms, from normalized frequency 0.15 onwards in the figure). An important characteristic of this type of excitation is producing time responses with better Gaussian distributions (less outlier, less transient effects).

Figure 21 shows a comparison of the same sensors (all at $27,000 \mathrm{ft}, \mathrm{Ma}=0.70$ ), with steady flight turbulence (MP13), pilot aileron/elevator stochastic (MP21) and wake turbulence excitation from the DLR-Falcon aircraft. As expected, flying in the wake turbulence of a preceding aircraft results in higher response levels, particularly in the higher frequency range (as can be seen from normalized frequency 0.15 onwards in the figure). On the other hand, random aircraft control excitation, though diligently executed by the pilot, injects little overall energy into the system.
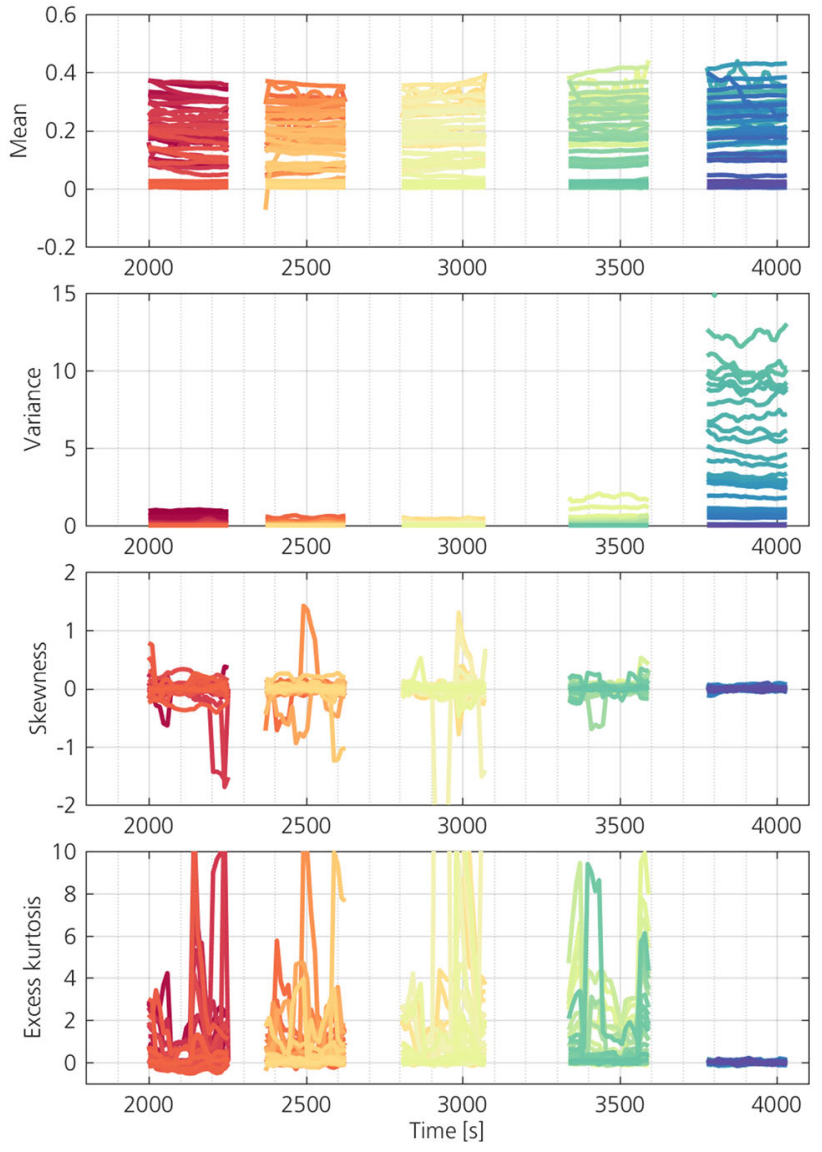

Fig. 19 Sample central moments of all accelerometers over time (MP11 $\div$ MP15)

\section{Loads measurement}

The aim of the in-flight loads measurement was to acquire data for the validation of the DLR loads processes. The conducted flight tests focused on the loads at the interface between the wing and the external store. The components of the external store are depicted in Fig. 22.

\subsection{Strain gauge positions}

The loads measurements were performed with strain gauges. On each hanger beam eight strain gauge bridges were 


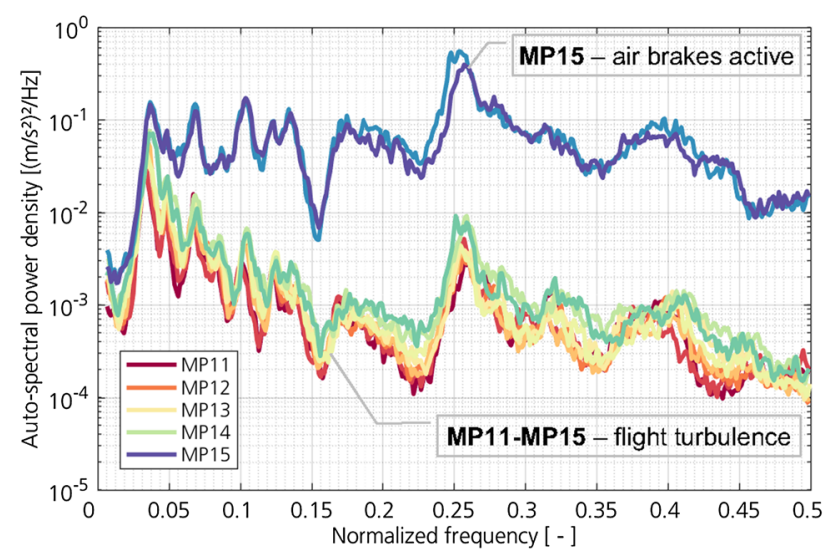

Fig. 20 Auto-power spectral densities of WING $+\mathrm{Z}$ accelerometers $(\mathrm{MP} 11 \div \mathrm{MP} 15)$

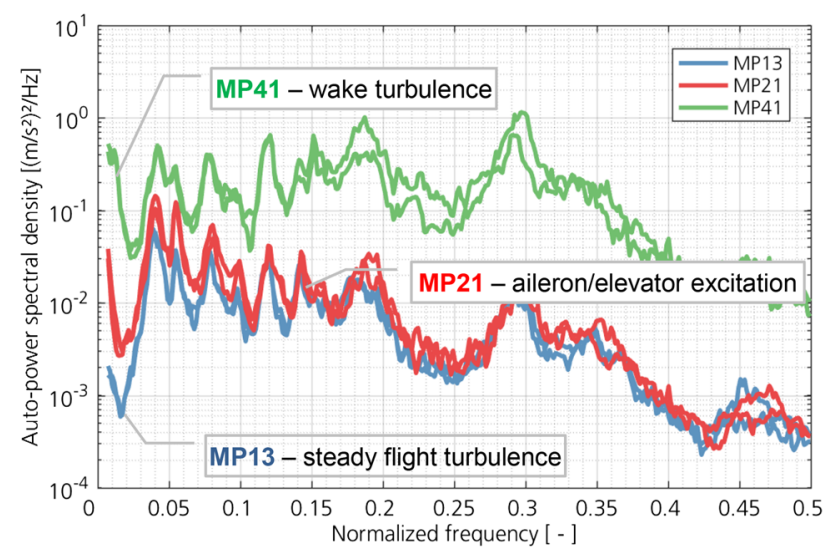

Fig. 21 Auto-power spectral densities of WING $+\mathrm{Z}$ accelerometers with different excitation types $(\mathrm{Ma}=0.70)$

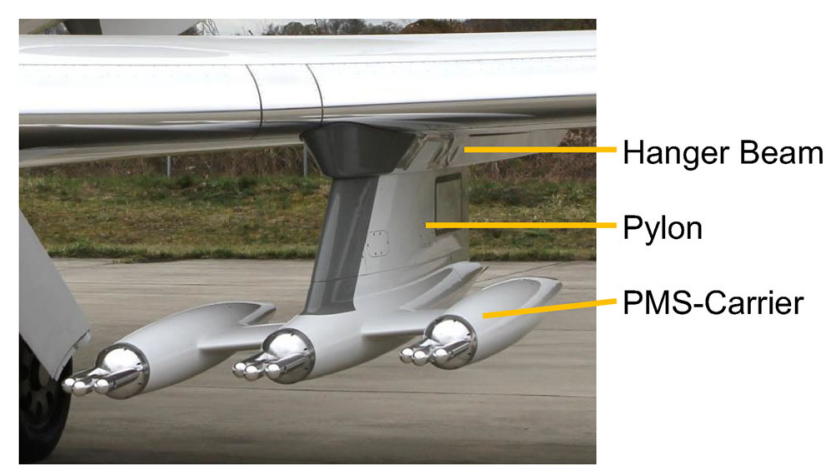

Fig. 22 Components of the utilized external store

installed. Six bridges could detect bending and two torsional deformation. The positions of the bridges have been determined with an FE-analysis to acquire measurable deformations under loading. Furthermore, two sensors of each bridge were placed on one side and the other two sensors on the opposite side of the hanger beam, as depicted in Fig. 5.

\subsection{Strain gauge calibration}

The strain gauges were calibrated by applying linearly independent loads on the PMS carrier. With sufficient applied loads-more than the number of bridges - the relationship between load and strain signal could be identified. This enabled the calculation of loads based on strain signals [16].

The first calibration of the strain gauges was performed at DLR in Göttingen (Fig. 23). For calibration the load was applied into the structure with the weight of sandbags. Weights up to $50 \mathrm{~kg}$ are attached to the force transmission points. To minimize the error due to hysteresis the applied loads are increased and decreased step by step and the strain signals are measured at each step. A typical strain response of a two-step load case is shown in Fig. 24.

The second calibration of the strain gauges was performed at DLR in Oberpfaffenhofen, where both PMS carriers were mounted on the wing station of the DLR HALO. In total, nine linearly independent loads were applied on each PMS carrier. From the strain signals transfer matrices were derived to calculate the actual applied loads. A picture of load application during the calibration is presented in Fig. 25.

\subsection{Loads measurement in flight}

Out of the five flight tests, the second was designated for manoeuvre loads. In that flight, several manoeuvres were flown in 21 flight conditions, which included seven different altitudes ranging from 12,000 to $35,000 \mathrm{ft}$ (3658 to $10,668 \mathrm{~m}$ ) with three different airspeeds each. The manoeuvres consisted of impulsive inputs on the steering yoke, accelerated rolls up to $\pm 45^{\circ}$ and pull ups up to $2 \mathrm{~g}$.

Initially, the idea for the flight test was to validate the numerical gust loads analysis with flight test data, since gust load cases are the relevant sizing loads for the interface, where the hanger beam is mounted to the wing. Unfortunately, the level of natural atmospheric disturbance

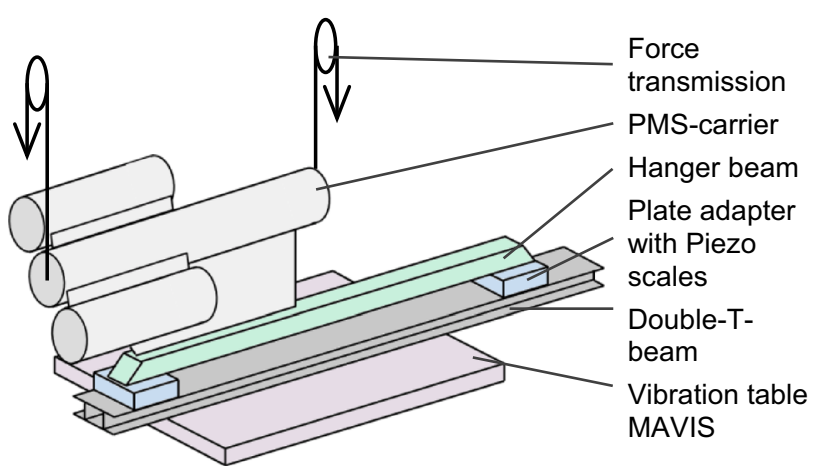

Fig. 23 Setup of the calibration on the MAVIS 


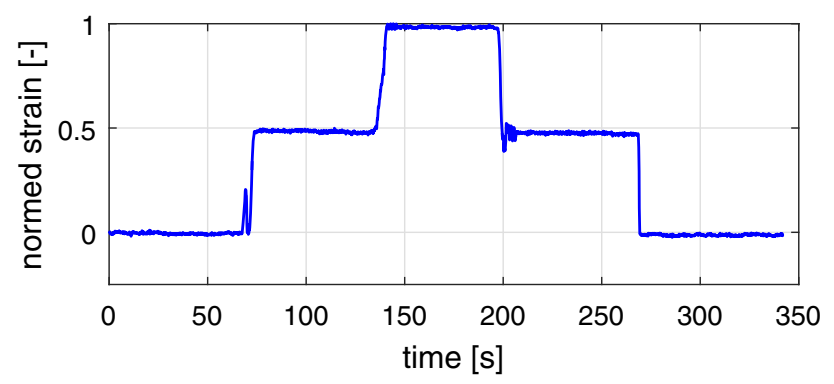

Fig. 24 Typical strain response of a two-step load transmission

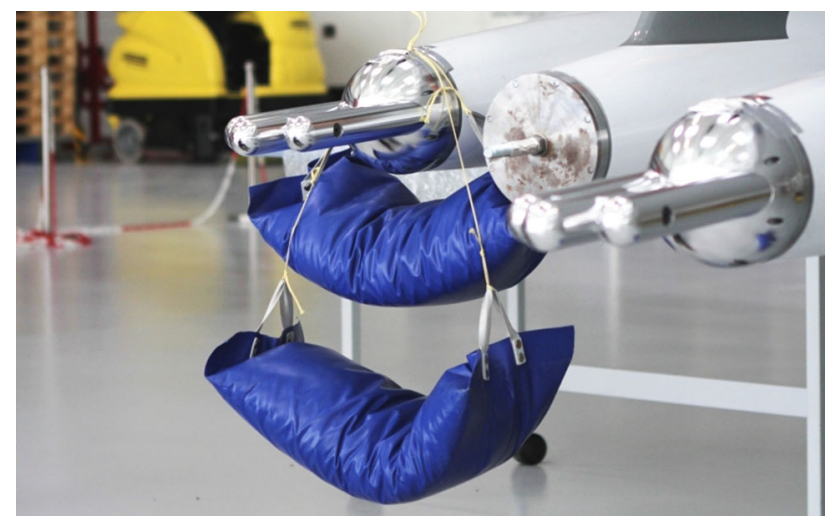

Fig. 25 Strain gauge calibration of a PMS carrier

was not high enough for sufficient natural excitation during the days of the test campaign. Thus, it was discussed to substitute atmospheric gust loads by the definition of manoeuvers with a similar frequency input for that flight. However, to achieve these defined manoeuvres, stick raps with a frequency of up to $14 \mathrm{~Hz}$ would be necessary. Such a procedure is difficult to perform manually with high precision. Besides, the control surface actuation systems would dampen the high frequency input to some degree. Instead, impulsive inputs on the steering system and low frequency manoeuvres as described above were considered as safer and easier to perform. A time history of a selected roll manoeuvre is shown in Fig. 26.

The normalized moments at the right PMS carrier during a pull up manoeuvre are presented in Fig. 27. In the first seconds it is apparent that the moments already fluctuate before the pitch angle changes. This indicates that the moments correlate in time with the acceleration of the aircraft. Further investigations of the loads based on the strain signals still have to be performed.

Beside the strain signals, data of atmospheric turbulence occurring during the flight is also collected. A first analysis showed that the power spectral density of the measured turbulence has a similar trend as a von-Kármán spectrum but with a smaller turbulence scale than $2500 \mathrm{ft}(762 \mathrm{~m})$. The latter value is defined in CS25 for loads calculations. A derived spectrum from the turbulence data and the

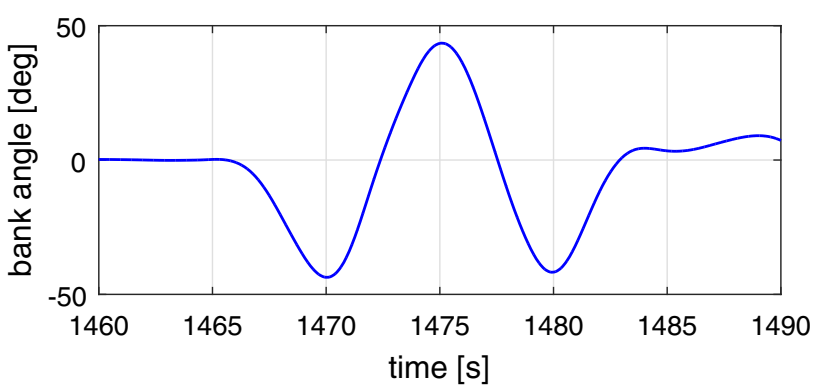

Fig. 26 Bank angle during a roll manoeuvre
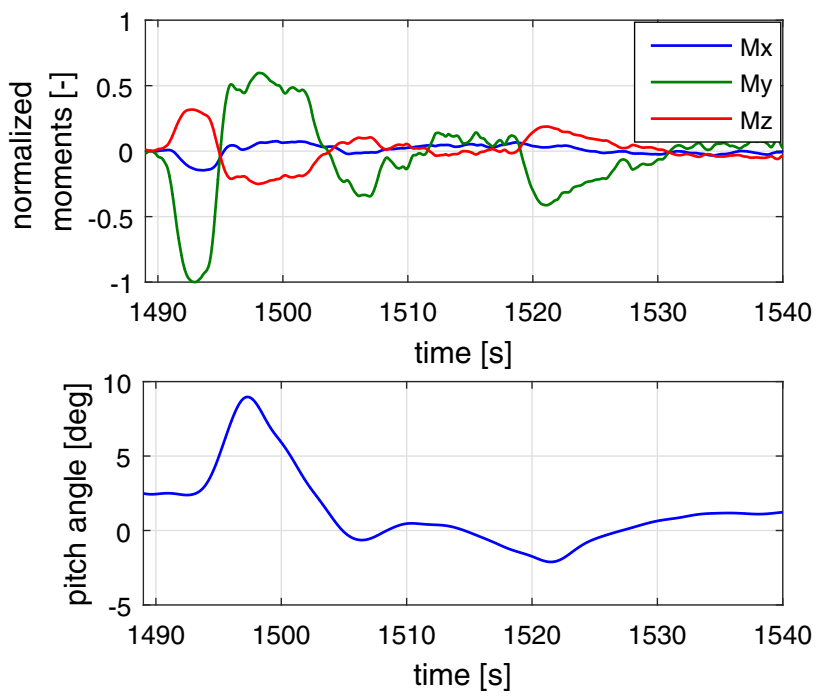

Fig. 27 Normalized moments during a pull-up manoeuvre (upper plot) the pitch angle during this manoeuvre (lower plot)

corresponding von-Kármán spectra are shown in Fig. 28. A smaller turbulence scale indicates that more energy is contained in the higher frequencies. This observation will be considered in further development of the DLR loads process.

\section{Conclusion and outlook}

The paper presents the preparation, execution and research scope of the iLOADS flight test campaign on DLR's research aircraft HALO. During flight test two major research aspects have been addressed: (1) online identification of the structural dynamical behaviour at different flight states and (2) in-flight loads analysis. Therefore, HALO was equipped with specially instrumented underwing-carriers, PMS.

New procedures for automated in-flight output-only modal analysis could be tested. Modal parameters (eigenfrequencies, mode shapes, and damping ratios) could be identified in almost real-time for each new flight condition. Every three seconds the complete modal data was available 


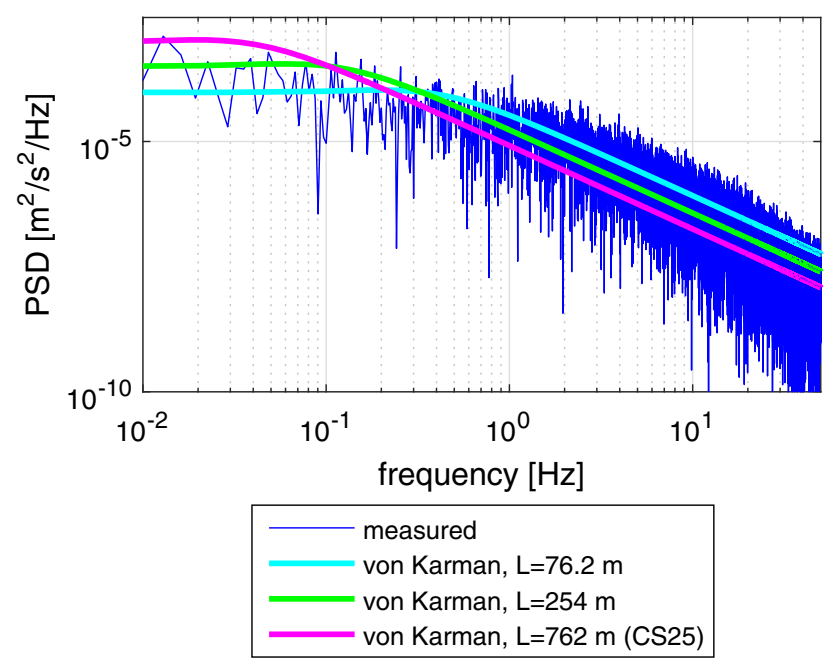

Fig. 28 Power spectral density of the measured turbulence, reference von-Kármán-spectra

and have been stored in a database. The data can be retrieved during the flight and the trend can be plotted over environmental parameters (altitude, speed...). On the basis of the raw data recorded in the time domain during the flights, it is possible to simulate the flights on a PC or to play back in real time in the laboratory. The aim is to improve the methods of the automatic output-only modal analysis, so that it is possible to quickly make decisions in the flight test about the vibration behaviour and to give a prediction for the next test point (e.g. the next higher flight speed or altitude).

Different excitation techniques from turbulence excitation, pulse-shaped control inputs from pilot, extended air brakes and wake vortices from a preceding aircraft have been used for the excitation of the aircraft. These excitation methods do not need external devices mounted to the aircraft or the access to an electronic flight control system. The influence of the different excitation techniques on the auto-power spectral densities has clearly been demonstrated.

The loads measurements are available for further evaluation and comparison with loads simulations. For this purpose, a loads model of HALO has been built up at DLR using an in-house aeroelastic modelling process supported by data from an extensive GVT performed by the Institute of Aeroelasticity in 2009, see [5].

Acknowledgements The authors would like to thank all members of the structural dynamics team and the aeroelastic design team for their dedication and commitment, namely, R. Buchbach, U. Füllekrug, H. Haupt, T. Klimmek, T. Meier, J. Schwochow and J. Springer. The authors also would like to thank the DLR flight test department in Oberpfaffenhofen, namely O. Brieger, S. Burwitz, S. Gemsa, S. Storhas and M. Hierle for perfect support especially during the flight test campaigns and A. Buschbaum and T. Wernsdörfer from the DLR design organisation. Finally the authors would like the DLR program directorate for the support within the DLR projects ALLEGRA and iLOADS.

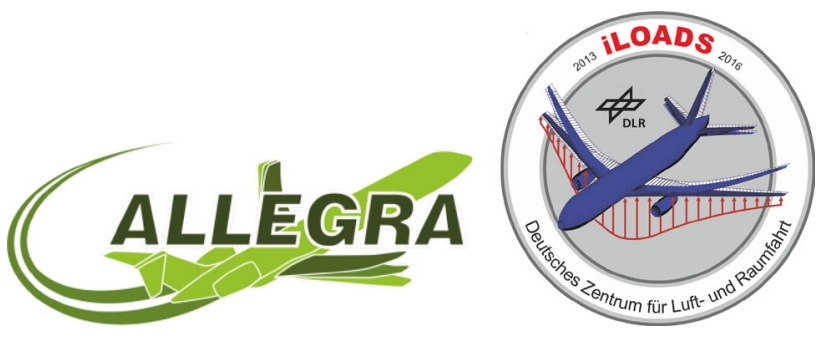

Open Access This article is distributed under the terms of the Creative Commons Attribution 4.0 International License (http://crea tivecommons.org/licenses/by/4.0/), which permits unrestricted use, distribution, and reproduction in any medium, provided you give appropriate credit to the original author(s) and the source, provide a link to the Creative Commons license, and indicate if changes were made.

\section{References}

1. Böswald, M., Göge, D., Füllekrug, U., Govers, Y.: A review of experimental modal analysis methods with respect to their applicability to test data of large aircraft structures. In: Proceedings of the International Conference on Noise and Vibration Engineering. Leuven, Belgium (2006)

2. Göge, D., Boeswald, M., Fuellekrug, U., Lubrina, P.: Ground vibration testing of large aircraft-state-of-the-art and future perspectives. In: Proceedings of the International Modal Analysis Conference. Orlando, Fl (2007)

3. Giclais, S., Lubrina, P., Stephan, C., Böswald, M., Govers, Y., et al.: New excitation signals for aircraft ground vibration testing. In: Proceedings of the International Forum on Aeroelasticity and Structural Dynamics. Paris, France (2011)

4. Govers, Y., Böswald, M., Lubrina, P., Giclais, S., Stephan, C., and Botargues, N.: Airbus A350xwb Ground vibration testing: efficient techniques for customer oriented on-site modal identification. In: Proceedings of the International Conference on Noise and Vibration Engineering. KU Leuven, Belgium (2014)

5. Krüger, W.R., Klimmek, T.: Definition of a comprehensive loads process in the Dlr Projekt Iloads. In: Proceedings of the Deutscher Luft- und Raumfahrtkongress 2016. Deutsche Gesellschaft für Luft- und Raumfahrt-Lilienthal-Oberth e.V., Braunschweig, Bonn (2016)

6. Jelicic, G., Schwochow, J., Govers, Y., Hebler, A., Böswald, M.: Real-time assessment of flutter stability based on automated output-only modal analysis. In: Proceedings of the International Conference on Noise and Vibration Engineering. KU Leuven, Belgium (2014)

7. Jelicic, G., Schwochow, J., Govers, Y., Hebler, A., Böswald, M.: Fast online monitoring and system identification for the application in the field of aeroelasticity. In: International Conference on Engineering Vibration 2015 (ICoEV2015): Ljubljana (Laibach), Slowenien (2015)

8. Jelicic, G., Schwochow, J., Govers, Y., Sinske, J., Buchbach, R., Springer, J.: Online monitoring of aircraft modal parameters during flight test based on permanent output-only modal analysis. In: 58th Aiaa/Asce/Ahs/Asc Structures, Structural Dynamics, and Materials Conference. American Institute of Aeronautics and Astronautics (2017) 
9. Hebler, A., Thormann, R.: Flutter prediction of a laminar airfoil using a doublet lattice method corrected by experimental data. In: Dillmann, A., et al. (eds.) New Results in Numerical and Experimental Fluid Mechanics X: Contributions to the 19th Stab/ Dglr Symposium Munich, Germany, 2014, pp. 445-455. Springer International Publishing, Cham (2016)

10. Mai, H., Hebler, A., Koch, S., Niehaus, K.: Aeroelastik Am Laminarflügel-Versuchsbericht Alf-5. Institut für Aeroelastik (2016)

11. Böswald, M., Govers, Y.: Taxi vibration testing —an alternative method to ground vibration testing of large aircraft. In: Proceedings of the International Conference on Noise and Vibration Engineering. KU Leuven, Belgium (2008)

12. Böswald, M., Govers, Y., Göge, D., Zhang, L.: Identification of modal parameters of aircraft during taxi. In: Proceedings of the International Modal Analysis Conference. The Printing House, Inc., Orlando (2008)
13. Dietz, G., Göge, D., Böswald, M., Govers, Y.: Verfahren Zur Gewinnung Von Daten Für Die Zulassung Eines Luftfahrzeugs, E.P. Office, Editor. Deutsches Zentrum für Luft- und Raumfahrt e.V., Germany (2008)

14. Böswald, M., Govers, Y.: Taxi vibration test: Die Anwendung Von Output-Only Modalanalyse Für Standschwingungsversuche Großer Flugzeuge. In: Proceedings of the 2. VDI-Fachtagung Schwingungsanalyse \& Identifikation. VDI Verlag $\mathrm{GmbH}$, Leonberg (2010)

15. Vacher, P., Jacquier, B., Bucharles, A.: Extensions of the Mac criterion to complex modes. In: Proceedings of the International Conference on Noise and Vibration Engineering. Leuven, Belgium (2010)

16. Skopinski, T.H., Aiken Jr., W.S., Huston, W.B.: Calibration of strain-gage installations in aircraft structures for the measurement of flight loads. NACA Report No. 1178, 1954 